\title{
Je crois que, je pense que : valeurs et variation dans un corpus oral diachronique
}

\author{
Nina Rendulić, Layal Kanaan-Caillol \\ Laboratoire Ligérien de Linguistique, UMR 7270, Université d'Orléans, France \\ nina.rendulic@univ-orleans.fr, layal.kanaan-caillol@univ-orleans.fr
}

Résumé. Nous étudions dans cet article deux structures parenthétiques, je crois que et je pense que, dans un sous-corpus d'ESLO (Enquêtes Sociolinguistiques à Orléans) constitué d'échantillons des enquêtes menées à 40 ans d'intervalle (ESLO1 et ESLO2). L'étude que nous proposons s'engage dans deux directions complémentaires. Nous observons premièrement, sur l'ensemble des données du sous-corpus, les variations des valeurs qui se sont révélées être communes aux deux structures. Les trois valeurs identifiées se situent sur une échelle qui interroge la prise en compte de l'interlocuteur allant de l'expression de l'univers mental du locuteur vers la sphère des croyances partagées.

A côté de l'approche qualitative, nous avons entrepris des requêtes quantitatives sur les données dans une perspective micro-diachronique, en comparant les deux structures jusque-là examinées conjointement. Les résultats montrent une forte progression des emplois de je pense que dans ESLO2 parallèlement à une baisse de ceux de je crois que, structure privilégiée dans ESLO1. Le changement observé est interprété à la fois en termes de déspécialisation de je pense que qui relaye je crois que dans certains de ces emplois et aussi en tant que conséquence directe du mouvement vers le domaine de la "pensée » au-delà des "croyances ». Finalement, ce changement semblerait attester un processus d'individualisation à l'œuvre dans l'expression de la modalisation.

\begin{abstract}
In this paper we study two parenthetical structures, je crois que and je pense que in a corpus extracted from ESLO (Sociolinguistic Survey in Orléans), which consists of samples of surveys carried out 40 years apart (ESLO1 and ESLO2). The study we propose is organized in two complementary topics. Firstly, we examine in our data the variation of values, which were shown to be shared by the two structures. Three identified values can be situated on a scale with regard to taking into account the interlocutor: those values oscillate between the expression of the locutor's mental universe and the sphere of shared beliefs.

Besides the qualitative approach, we undertake quantitative surveys on our data in a micro-diachronic perspective, in order to compare the two structures we had studied jointly. The results show a significant increasing of the use of je pense que in ESLO2 together with a decreasing use of je crois que, structure which is preferred in ESLO1. We interpret this alternation as a result of two factors: firstly, as a despecialisation of je pense que which relays je crois que in some of its uses, and secondly, as a direct consequence of the motion towards the domain of "thoughts", beyond "beliefs". Finally, this motion seems to attest a certain process of individualization existing in the expression of the modalization.
\end{abstract}




\section{Introduction}

Nous nous proposons dans cet article d'étudier, dans un corpus oral d'environ 70 heures (850 000 mots), deux configurations verbales introduisant une complétive, je crois que et je pense que. Leur désignation en tant que « recteurs faibles » (Blanche-Benveniste 1989) ou « verbes parenthétiques » (Urmson 1952, Andersen 1996) traduit un premier axe d'analyse associé à ces structures, fréquemment en français oral. Il s'agit de rendre compte de leur particularité syntaxique: ces verbes peuvent à la fois régir une subordonnée complétive ("je pense qu'on se sert d'Orléans comme un quartier de Paris») et figurer dans une construction sans «que », en incise ("il est je crois en deuxième année ») ou postposés ( y en a au moins cinq boulangeries je crois ») à une proposition. A partir de ces deux moyens de construction syntaxique, Apothéloz (2003) étudie les propriétés syntaxiques de la rection faible, afin d'en proposer un classement qui traduirait une disjonction sémantico-énonciative ${ }^{1}$ à l'intérieur du système construit par ces verbes. Au nombre de leurs propriétés syntaxiques, il dénombre la non-pronominalisation de la complétive, l'instabilité morpho-phonologique de que, l'insensibilité face à la négation et à l'interrogation, la limitation à certains temps et personnes grammaticales et le déplacement du topique vers la complétive. Cette catégorisation syntaxique met en évidence deux paradigmes énonciatifs : celui où croire et penser gardent leur sens plein et ne sont pas concernés par les propriétés syntaxiques des recteurs faibles («j'ai pensé à toi hier»), et celui qui correspond aux critères énoncés («je pense que c'est sur la cinq »).

A partir du constat de ce double paradigme sémantico-énonciatif dans l'interprétation des constructions je crois que et je pense que, notre étude s'engage dans deux directions. D'une part, il s'agira de confronter les occurrences de notre corpus aux valeurs et aux emplois déjà attestés dans la tradition des études sur les verbes « recteurs faibles », afin de proposer une réinterprétation énonciative des liens entre les valeurs véhiculées par ces verbes. Il semblerait en effet que la disjonction des emplois attestés de ces deux configurations puisse se définir sur une échelle qui se dessine entre l'univers mental du locuteur et la circulation des croyances sociales, tout en se précisant dans l'image de l'interaction.

D'autre part, nous adopterons une approche variationniste en micro-diachronie : à quarante ans d'intervalle, nos données montrent une hausse considérable de la fréquence des constructions je pense que parallèlement à une baisse de fréquence de je crois que. L'analyse qui s'en suivra proposera une interprétation de ce changement en termes sémantico-cognitifs, indissociables des paramètres interactifs et sociaux.

\section{Les données}

L'analyse porte sur des occurrences je crois que / je pense que attestées dans le corpus Enquêtes Sociolinguistiques à Orléans (désormais ESLO). Il s'agit d'un grand corpus de documents sonores qui regroupe deux enquêtes menées à 40 ans d'intervalle : la première qui date des années 1968-1974 a été menée par une équipe d'universitaires franco-britannique, ESLO1, et la deuxième, engagée en 2008, est portée par une équipe de chercheurs du Laboratoire Ligérien de Linguistique, ESLO2 (cf. Baude \& Dugua 2011, Eshkol-Taravella \& al. 2012). Réunis, ESLO1 et ESLO2 forment une collection de 700 heures d'enregistrement, 10 millions de mots, dont 5 millions mis à disposition actuellement ${ }^{2}$. 
Il s'agit là d'un corpus variationniste, qui offre un accès aux variations micro-diachroniques mais aussi aux variations diaphasiques, grâce aux différentes situations de collecte (entretiens, conférences, commerces, micro-trottoir, repas, etc.), et diastratiques, avec une classification des locuteurs selon l'échelle d'Alix Mullineaux ${ }^{3}$ pour ESLO1 et selon les catégories socio-professionnelles définies par l'INSEE pour les locuteurs d'ESLO2 ${ }^{4}$.

Pour notre étude, nous sommes parties d'un sous-corpus d'ESLO constitué par L. Abouda et M. Skrovec en vue d'une approche micro-diachronique de l'expression du futur (cf. Abouda \& Skrovec (2015)). Constitué à parts égales d'enregistrements d'ESLO1 et d'ESLO2 en termes de nombre d'heures, le souscorpus garantit aussi, dans la mesure du possible, un équilibrage diastratique ${ }^{5}$ entre les deux enquêtes et à l'intérieur de chacune d'elles.

Pour cette première approche des constructions qui nous intéressent et en vue d'une comparabilité microdiachronique optimale, nous avons sélectionné dans ce sous-corpus les entretiens uniquement ${ }^{6}$, ce qui correspond à 68 heures d'enregistrements (34 heures d'ESLO1 / 34 heures d'ESLO2) pour un total de 850000 mots. L'idée étant de repérer les différentes valeurs des unités dans les entretiens et de confronter la typologie, dans un deuxième temps, à d'autres situations.

Dans la présente étude, bien que les requêtes aient porté sur les verbes croire et penser, nous nous limitons aux constructions je + crois/pense + que et laissons de côté, pour une étude ultérieure, les occurrences je crois/je pense en tant que marqueurs discursifs.

L'exploitation de données s'est faite avec le logiciel d'analyse textométrique TXM qui permet d'exporter les résultats des requêtes sous forme de tableaux CSV. Chacune des occurrences répertoriées a donc fait l'objet d'une annotation qui a été accompagnée selon les cas de précisions notamment en lien avec des indices contextuels. Ainsi nous avons travaillé sur 474 occurrences de je crois que et sur 417 occurrences de je pense que.

\section{Croire (que) / penser (que) : emplois et valeurs}

D'un point de vue syntaxique, les verbes croire et penser, comme tous les verbes dits «parenthétiques » ou « recteurs faibles », apparaissent dans trois types d'emplois : emplois propositionnels, emplois suivis de que- $p$ et emplois en incise médiane ou finale. Ces différents types d'emplois syntaxiques induisent des différences importantes du point de vue sémantique observées en comparant les emplois propositionnels aux deux autres emplois.

Ainsi, les études consacrées aux deux verbes, appréhendés notamment dans le cadre d'approches contrastives et traductologiques français/anglais (à l'écrit, Doro-Mégy 2008, à l'oral, Mullan 2010, 2012), se rejoignent dans la description sémantique de chacun de ces verbes. Sur le sémantisme de je pense Mullan (2012) note qu'il exprime :

\footnotetext{
an opinion based on reflection, where the speaker wishes to positively assert or claim a particular stance towards a proposition. The verb penser ('to think') is connected to cogitation and reflection $[\ldots]$ (p. 10)
}

Alors que je crois exprime les croyances, les convictions et évoque l'idée d'acceptation liée à la foi en quelque chose ; «With this state of mind there is no need for reflection » (Mullan 2010 : 137).

Ces différences évidentes entre les sens pleins des deux verbes sont, dans les emplois modaux, réduites à des différences dans le positionnement l'échelle de la certitude. En effet, Kerbrat-Orecchioni (1980 : 113) suggère que croire exprime plus d'incertitude que penser.

Or les données de notre corpus montrent que, malgré les spécificités sémantiques de chaque verbe, les emplois modaux de je crois que et je pense que, considérés du point de vue des locuteurs, sont interchangeables (cf. infra, exemples 9, 10 et 11). 


\subsection{Je crois, je pense : valeurs des emplois propositionnels}

Le corpus ESLO, dans son intégralité, atteste de très peu d'occurrences des emplois propositionnels. Pour croire, nous relevons 6 occurrences de je crois (ESLO1 et ESLO2 confondus). Ces emplois expriment l'adhésion du locuteur à des sciences ou des domaines de connaissance (exemple 1) ou encore à des valeurs ou à une pratique (exemples 2 et 3 ).

Exemple 1 (ESLO1_ENT_079_je crois_01:03:20)

Iz248 : [...] $]^{7}$ je fais de la graphologie enfin je fais beaucoup moins maintenant mais je crois à la graphologie [rire] j'y attache une grande importance

Exemple 2 (ESLO1_ENT_017_je crois_00:19:10) : en parlant du scoutisme

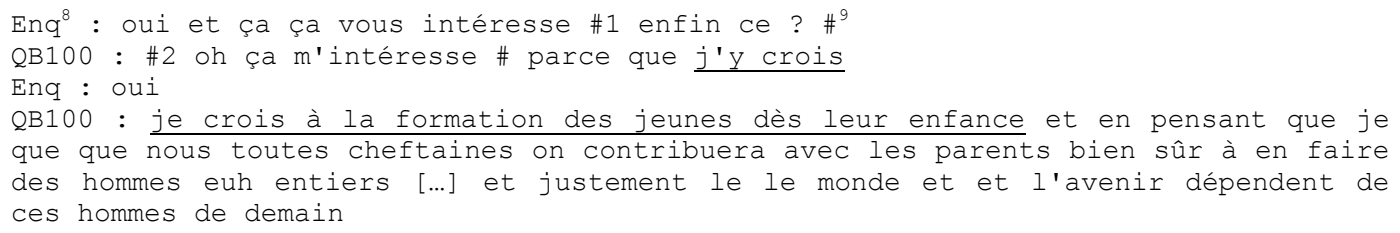

Exemple 3 (ESLO2_ENT_1005_je crois_01:00:20)

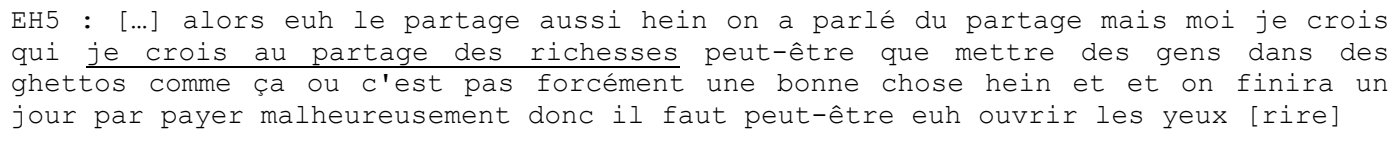

Un seul exemple atteste de l'emploi de croire qui pointe l'existence comme dans «croire au Père Noël » :

Exemple 4 (ESLO1_ENT_072_je crois_00:23:50)

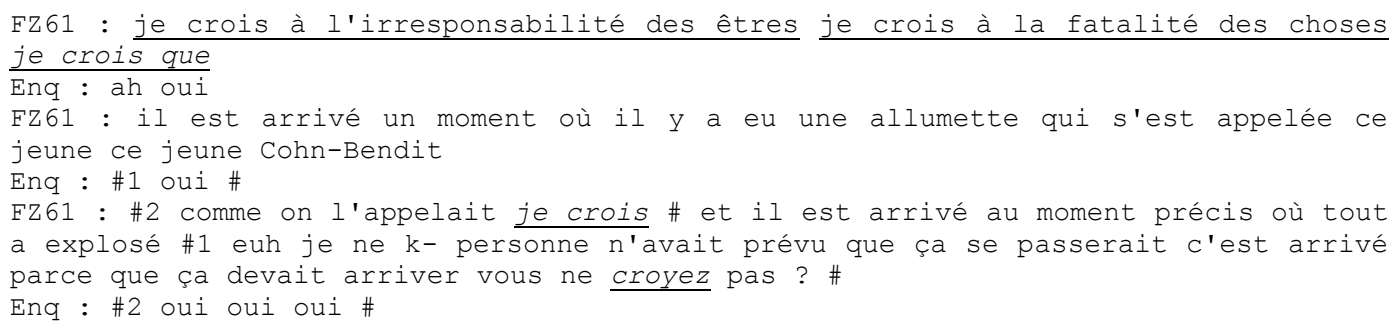

En abordant les événements de mai 68 - l'enregistrement datant d'avril 1969 -, la locutrice (professeur de piano, 62 ans), énonce sa croyance sur le modèle du Credo, une liste dont le troisième élément quitte le solennel et se raccroche au genre discursif attendu. Il est intéressant de relever dans cet exemple la coapparition des trois types d'emplois syntaxiques de je crois (propositionnel, + que et en incise) et une dernière occurrence à la deuxième personne qui cherche à vérifier la dimension commune de l'univers de croyance évoqué.

Pour penser, nous n'avons relevé aucune occurrence de l'emploi propositionnel « absolu » illustré dans le fameux «je pense donc je suis ». Seul un emploi avec « on » rencontré dans ESLO1 y correspond ${ }^{10}$. Aucun emploi non plus de penser avec un complément d'objet direct comme dans «penser la communication».

Par ailleurs, trois valeurs relatives aux emplois propositionnels de je pense ont été identifiées, elles correspondent toutes à la construction « je pense à ». La première est illustrée dans l'exemple 5 : 
Exemple 5 (ESLO1_ENT_072_je crois_00:23:50)

FZ61 : mais même ma fille aînée avec ses tournées de concerts j'ai souvent je suis navrée quand je pense à ma petite fille qui reste seule confiée à [...]

Il s'agit là d'un emploi dans le sens de «évoquer dans son esprit », cependant, contrairement aux deux autres emplois/valeurs, le présent de « je pense » ne renvoie pas à un acte en cours, il relève de ce que les grammairiens appellent le présent d'habitude. Cette valeur de « je pense », qui actualise un ressenti/une émotion serait difficilement repérable en discours avec un présent d'actualité, elle n'apparaitrait que dans le cas particulier où le locuteur fait état de son activité cérébrale. Une telle occurrence supposerait un silence en amont.

La deuxième valeur que revêt je pense propositionnel, et qui partage avec la première l'emploi d'un présent «a-temporel », a été repérée dans des constructions du type faudra que je pense /faudra penser, fais-moi penser où penser au contact de «falloir » et de «faire », à côté d'autres éléments contextuels, prend la valeur de rappeler.

Exemple 6 (ESLO2_ENT_1049_je pense_01:30:29)

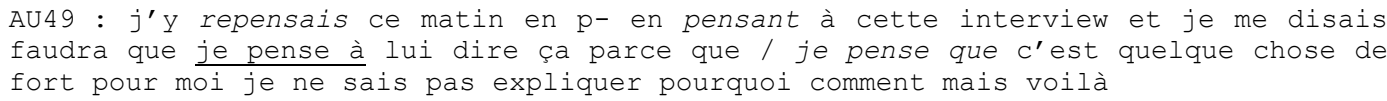

Bien que l'occurrence soulignée soit celle qui nous intéresse ici, on ne peut que noter les trois autres emplois de «penser» dans un seul tour de parole, une prouesse linguistique à la manière d'un virelangue !

La troisième valeur émerge de l'emploi de je pense dans le cadre d'une explication par extension, dans le sens de «par exemple », le locuteur décrit ainsi le mécanisme sous-jacent à son propos («si je dis ça c’est parce j'ai une expérience précise ») :

Exemple 7 (ESLO1_INTPERS_414_je pense_00:50:10)

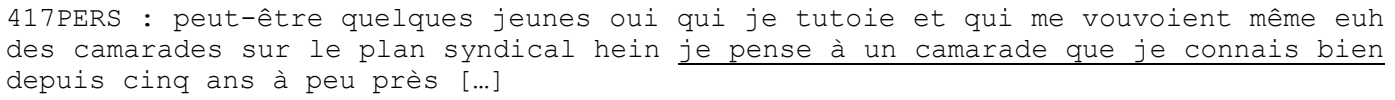

Exemple 8 (ESLO2_DIA_1225_je pense_00:50:10)

DIA 1225 : des émissions à thème m’intéressent je pense à certaines émissions d'Arte euh qui sont intéressantes

A côté de ces quelques emplois propositionnels attestés dans le corpus, emplois que nous appelons aussi «pleins », des verbes je crois et je pense, nous retrouvons ces derniers, de manière beaucoup plus fréquente en $\mathrm{V}+$ que où le propos (topique) n'est plus dans penser et croire mais dans la complétive.

Est-ce que je me décris croyant quand je dis je crois (que...) ? Sûrement non. L'opération de pensée n'est nullement l'objet de l'énoncé ; je crois (que...) équivaut à une assertion mitigée. En disant je crois (que...), je convertis en une énonciation subjective le fait asserté impersonnellement, à savoir le temps va changer, qui est la véritable proposition. (Benveniste, 1966, p. 264)

Ces constructions parenthétiques intronisent croire et penser dans l'univers de la modalisation, celui plus particulièrement de la modalité épistémique.

Malgré les différences des contextes d'emplois de je crois que et je pense que liées à l'appartenance du premier au champ de la connaissance et du second à celui du jugement (cf. Martin, 1988), on remarque que ces emplois « recteurs faibles » partagent une même échelle de valeurs en discours (cf. 3.2 cidessous). Cela est d'autant plus intéressant à observer quand ce sont les locuteurs eux-mêmes qui le 
prouvent : un seul locuteur (exemples 9 et 10) ou dans des énoncés identiques de deux locuteurs différents (exemples 10 et 11 )

Exemple 9 (ESLO1_ENT_029_je pense que/je crois que_00:42:17)

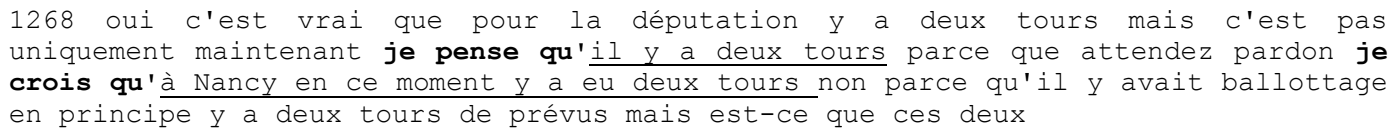

Exemple 10 (ESLO1_ENT_052_je pense que/je crois que_00:39:32)

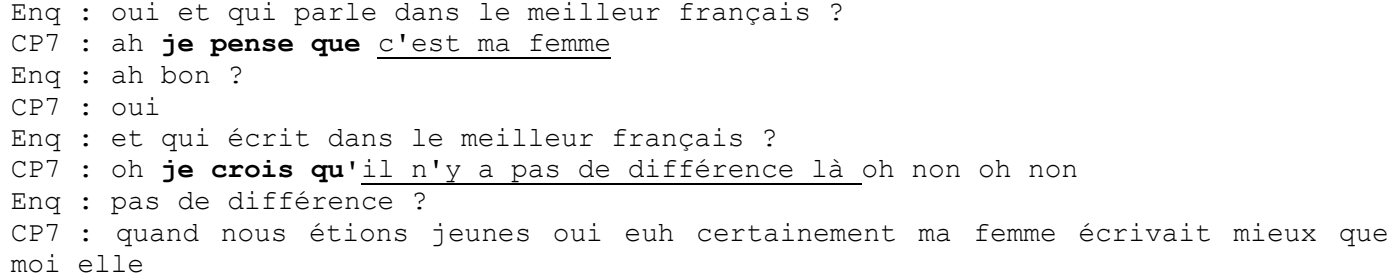

Exemple 11 (ESLO1_ENT_001_je crois que_00:55:48)

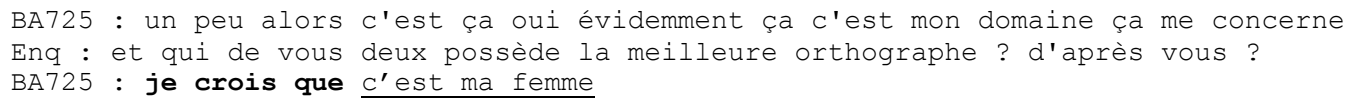

Pour les exemples 9 et 10, les locuteurs emploient je crois que et je pense que dans un même contexte et leur permutation n'induirait pas de changement de sens. Cela est d'autant plus flagrant à observer quand on compare les exemples 10 et 11 , où dans des contextes fortement comparables, les locuteurs emploient indifféremment les deux formes.

C'est à partir de ces constats (point de vue des locuteurs) que nous abordons je crois que et je pense que conjointement en nous intéressant aux valeurs qui se sont révélées communes aux emplois de chacune des deux formes (point de vue de linguistes, non moins locuteurs).

\subsection{Je crois que, je pense que : trois valeurs épistémiques}

Nous appellerons valeurs modales ou épistémiques celles qui sont associées aux structures je crois que et je pense que lorsque celles-ci sont employées en tant que verbes recteurs faibles. La plupart des études existantes sur les structures actualisant les verbes recteurs faibles privilégient soit une approche syntaxique - certes indispensable pour délimiter le champ de la rection faible, mais qui ne rend pas compte de la systématicité et de la distribution des valeurs qui leurs sont sous-jacentes - soit celle où les valeurs sémantiques des structures ne sont pas, ou peu, mises en corrélation ou étudiées en contexte. Or il $\mathrm{y}$ a tout intérêt à analyser l'échantillonnage des valeurs relativement au contexte interlocutif, c'est-à-dire en associant les intentions du locuteur au contexte communicationnel. Une telle démarche, que nous avons adoptée dans cette étude, permet d'identifier les diverses valeurs modales non seulement per se mais aussi et surtout en extension, en opposant les catégories de valeurs afin de délimiter leurs frontières externes. Observons les trois exemples suivants, extraits de notre corpus :

Exemple 12 (ESLO2_DIA_003_je pense que_00:41:46)

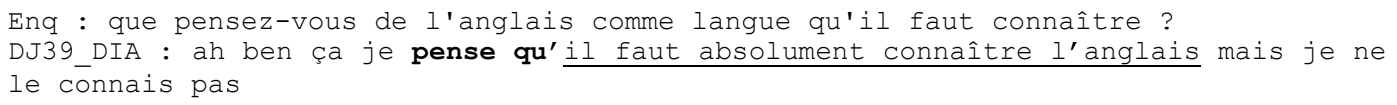


Exemple 13 (ESLO1_ENT_160_je crois que_00:53:11)

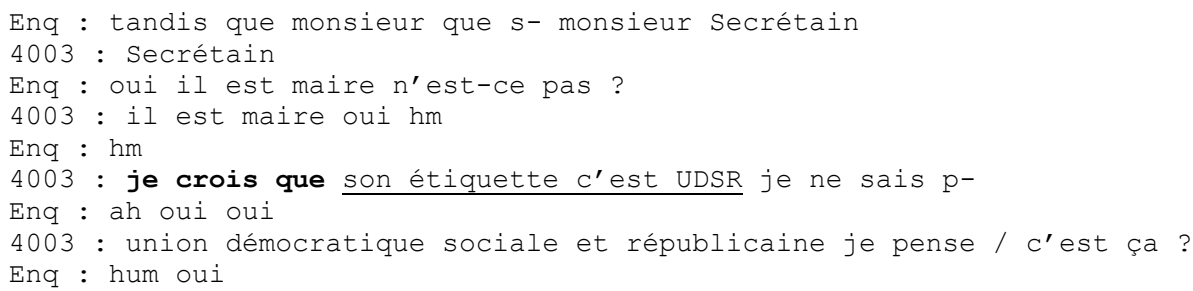

Exemple 14 (ESLO1_ENT_001_je crois que_00:04:48)

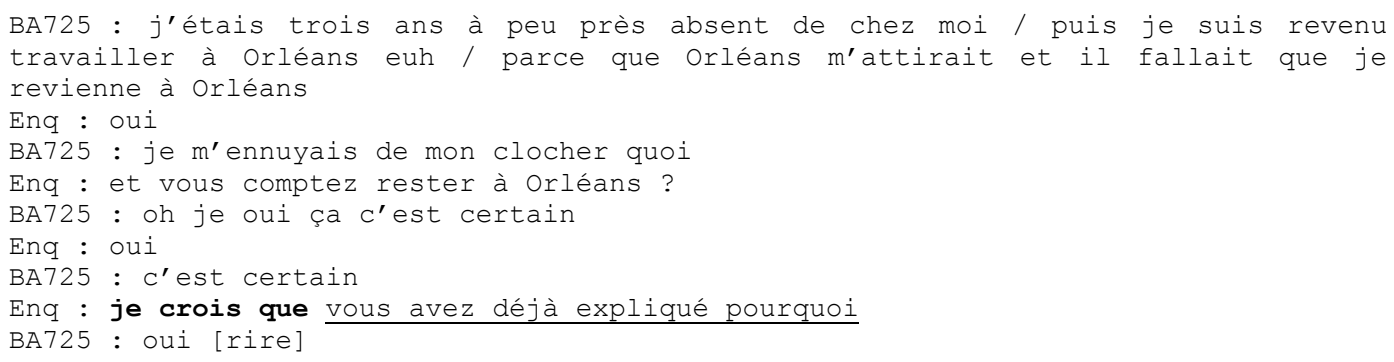

Dans son article sur les verbes recteurs faibles dans les propositions rectrices de complétives, Apothéloz (2003) distingue deux valeurs modales associées aux propositions rectrices: celle de «modalité incidente » et celle d' " atténuation socio-interactionnelle ». La première valeur module «épistémiquement la validité [d'une proposition]» (Apothéloz, 2003 : 249), alors que la seconde s'associe à un «dispositif d'atténuation permettant de formuler une requête d'une façon socialement acceptable et donc "polie". » (Apothéloz, 2003 : 253). Autrement dit, la valeur de «modalité incidente» s'actualise dans des structures à verbes recteurs faibles lorsque la proposition rectrice exprime le point de vue du locuteur, modulant l'assertion énoncée dans la complétive, qui porte le contenu informationnel de l'énoncé dans son ensemble. Cette valeur se retrouve dans notre exemple 12: l'énoncé il faut absolument connaître l'anglais est modulé selon le point de vue de DJ3 DIA. Par conséquent, d'une part l'affirmation est subjectivisée par l'opinion du locuteur, et d'autre part elle est modalisée : il ne s'agit pas d'affirmer ou de relativiser la valeur de la vérité exprimée par la complétive, mais de délimiter sa portée, autrement dit, de la modaliser selon le point de vue du locuteur.

La seconde valeur de structures à verbe recteur faible repérée par Apothéloz (2003) s'illustre dans notre exemple 14 : je crois que n'exprime pas le point de vue du locuteur quant au contenu propositionnel de la complétive, mais module, atténue une information afin qu'elle ne paraisse pas « autoritaire », «tranchante » ou trop «directe». Ceci se confirme en contexte. Le locuteur BA725 explique les raisons pour lesquelles il souhaite rester à Orléans, avant d'affirmer sa décision suite à la question posée par l'enquêteur (et vous comptez rester à Orléans? / oh je oui). Celui-ci n'a donc pas besoin de demander pourquoi, mais il l'affirme, tout en modulant ses propos par je crois que.

A part ces deux valeurs de structures à verbes recteurs faibles qu'explique Apothéloz (2003), dans notre corpus nous avons pu en identifier et définir une troisième, qui est illustrée par l'exemple 13. Ici, je crois que est à observer relativement à d'autres marqueurs modaux en contexte : je ne sais p-, je pense, c'est ça? Ceux-ci partagent une caractéristique commune : ils expriment l'incertitude.

Désormais seront détaillées les caractéristiques de chacune des trois valeurs isolées à partir des données de notre corpus. Il importe de signaler que ces trois valeurs s'actualisent aussi bien avec croire qu'avec penser et que leur répartition au sein des deux structures reste stable. Nous concluons donc que ce n'est pas le sémantisme des deux verbes, mais la portée interactionnelle de la structure [je] + [(croire/penser) $P Z]+[q u e]$ dans son ensemble qui module les énoncés en complétive en leur accordant trois effets de sens différents. 


\subsubsection{Valeur d'argumentation subjective}

Les séquences je crois que / je pense que peuvent interroger le point de vue du locuteur quant au contenu propositionnel des énoncés dans la complétive. Déjà démontrée par l'exemple 12, la valeur associée à ces séquences concerne l'expression subjective du locuteur qui émet son avis, son jugement, ses convictions intimes sur le contenu propositionnel exprimé par la complétive.

Dans notre corpus, cette valeur d'argumentation subjective s'associe surtout aux formes je pense que: environ $75 \%$ des occurrences avec penser dans les deux sous-corpus, ESLO1 et ESLO2, obtiennent cette valeur, contre environ $45 \%$ dans les sous-corpus pour les structures je crois que. Cette constatation sera détaillée dans la partie 4 de la présente étude: notons que le verbe penser semble être davantage spécialisé pour exprimer ce qui est propre au locuteur, donc sa subjectivité.

Quel est le type de contenu propositionnel que le locuteur module par je pense que / je crois que à valeur d'argumentation subjective ? Le cas le plus fréquent se rapporte aux évaluatifs : par les structures je crois que / je pense que, le locuteur émet son avis, son opinion sur «le monde qui l'entoure ». Les deux exemples suivants montrent certains de ces emplois.

Exemple 15 (ESLO1_ENT_003_je crois que_00:11:41)

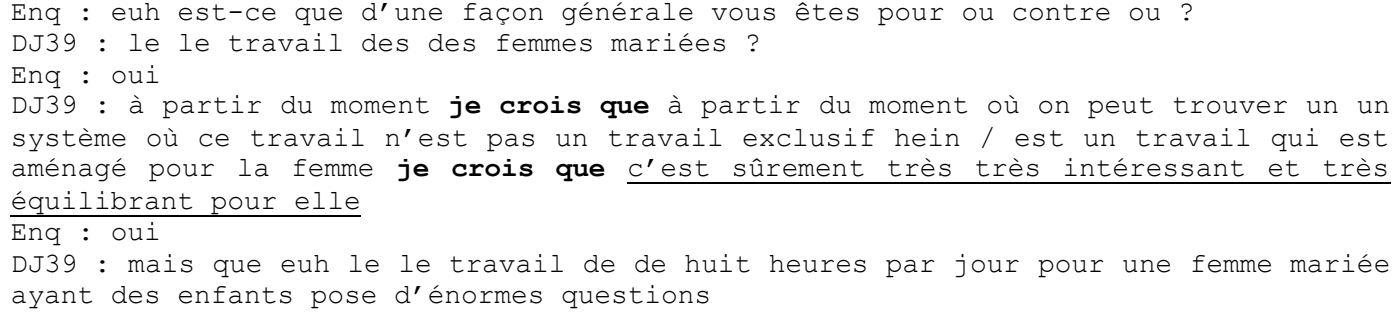

Exemple 16 (ESLO2_ENT_1049_je pense que_00:11:38)

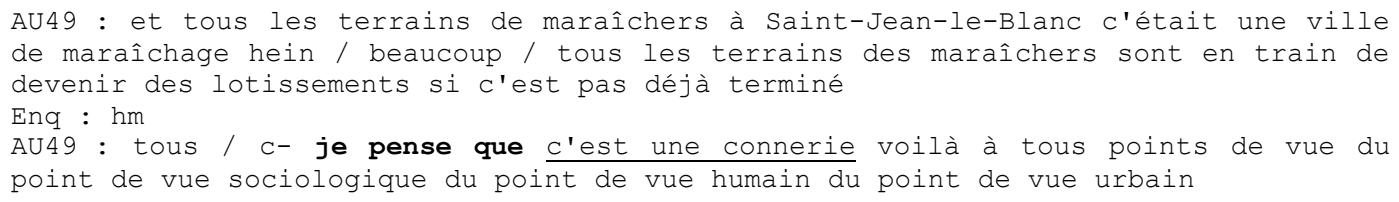

Ce type d'emploi évaluatif se caractérise par un haut taux de présentatifs ( $c$ 'est, il y a), de verbes au présent dans la complétive marquant «l'intemporalité » (dont notamment les verbes modaux falloir et pouvoir, cf. Exemple 12) ainsi que de certains adverbes ou de substantifs à valeur pragmatique d'évaluation. Notons-en quelques-uns : invivable, important, dramatique, faisable, très bien, vachement utile, du temps perdu, une grave erreur, quelque chose de fort.

Ce qui caractérise par excellence les occurrences de je crois que / je pense que ayant la valeur d'argumentation subjective, c'est le double «retour sur soi » : aux énoncés qui par eux-mêmes traduisent une évaluation, un point de vue de celui qui les énonce (c'est intéressant / c'est une connerie), le locuteur ajoute une seconde échelle de la subjectivité qui se cristallise dans le marquage par je crois que / je pense que. Doublement subjectifs, ces énoncés semblent exclure, ou au moins ignorer la figure de l'interlocuteur dans le solipsisme de leur énonciation. Nous y reviendrons en 3.4.

\subsubsection{Valeur d'incertitude}

Par rapport à la classification d'Apothéloz (2003), nous avons pu isoler une valeur supplémentaire véhiculée par je crois que / je pense que, visible dans l'exemple 13. Les structures à verbes recteurs faibles expriment l'incertitude du locuteur quant à la validité du contenu propositionnel enchâssé : 
reformulables par il me semble ou j'ai l'impression...mais je ne suis pas sûr, ces énoncés véhiculent divers degrés de la non prise en charge par le locuteur des propos qu'il énonce.

Exemple 17 (ESLO1_ENT_150_je crois que_00:34:11)

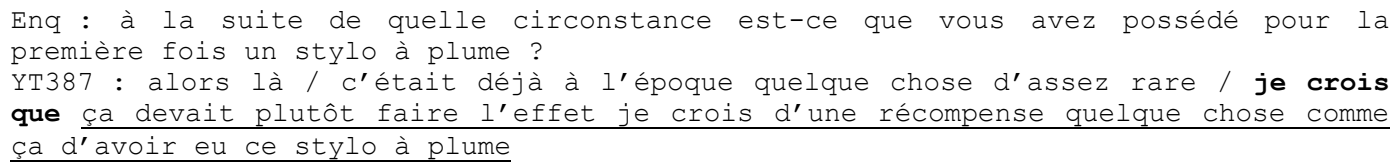

Dans cet exemple, le locuteur ne parvient pas à se souvenir à quelle occasion il a eu son premier stylo à plume : sans en être certain, il suppose qu'il s'agissait d'une récompense. L'incertitude du locuteur, véhiculée par je crois que, se confirme par d'autres marques : le verbe devoir, la répétition de je crois et le syntagme qui relativise le mot récompense, quelque chose comme ça.

Il est fréquent que les occurrences de je crois que / je pense que à valeur d'incertitude soient accompagnées d'autres marques cotextuelles qui confirment et rehaussent cette non prise en charge de propos exprimés par la complétive. L'exemple suivant montre le degré de «précaution » prise par le locuteur dans l'expression de la non prise en charge de ses propos.

Exemple 18 (ESLO1_ENT_115_je pense que_00:26:46)

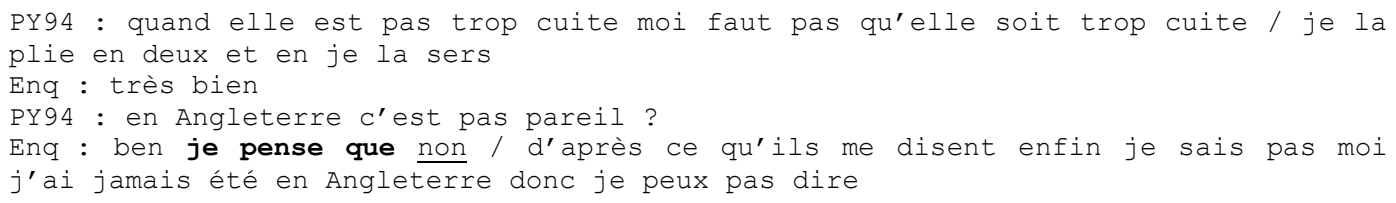

Dans cet extrait, la locutrice PY94 détaille la façon dont elle fait une omelette. A la fin de son explication, certainement étonnée par la question, elle demande s'il y a une autre manière de faire des omelettes en Angleterre, croyant que l'enquêteur est d'origine britannique, comme la plupart de chercheurs-enquêteurs dans l'enquête ESLO1. Celui-ci répond négativement, tout en modulant son affirmation : non seulement il pense que non, il n'est pas certain, mais il surmarque son incertitude par la suite, d'abord par une assertion autre, d'après ce qu'ils me disent, ensuite par un énoncé exprimant explicitement son incertitude, je sais pas, argumenté par le fait qu'il n'est jamais allé en Angleterre, et enfin par l'énoncé je peux pas dire, par lequel il décline toute responsabilité énonciative pour le premier non.

Propre aux occurrences à valeur d'incertitude, ce surmarquage accentue la non prise en charge du contenu propositionnel de la complétive. En outre, ces je crois que / je pense que interrogent la situation du locuteur au sein de la situation de communication : le «dire vrai », c'est-à-dire l'affirmation de son incertitude, de son non-savoir, est en interaction une force. D'une part, il représente l'humilité du locuteur qui marque ses limites, et d'autre part il évite des conflits potentiels dus aux malentendus entre les interlocuteurs.

\subsubsection{Valeur d'atténuation et de certitude}

La troisième valeur de je crois que / je pense que isolée au sein des occurrences de notre corpus recouvre partiellement celle qu'Apothéloz (2003) appelle «valeur d'atténuation socio-interactionnelle »: elle se rapporte aux occurrences qui pourraient, en interaction, être perçues comme trop directes, trop autoritaires. Quand bien même le contenu propositionnel dans la complétive ne ferait pas, pour le locuteur, l'objet ni d'une incertitude ni d'un avis explicite, sa modulation par je crois que / je pense que s'avère être un outil de gestion des relations interlocutives. Au-delà de la politesse interactionnelle que je crois que / je pense que accordent aux énoncés dont la véracité n'est pas mise en doute, une autre nuance d'emplois se retrouve parmi les occurrences de notre corpus.

Dans cette perspective, nous dirons que la valeur d'atténuation n'est qu'une des manifestations du sémantisme inhérent à cette configuration, qui s'actualise par deux valeurs ayant en commun la certitude, 
pour le locuteur, quant à la validité du contenu propositionnel de la complétive. Expliquons cette hypothèse sur les deux exemples suivants :

Exemple 19 (ESLO2_ENT_1041_je pense que_00:00:37)

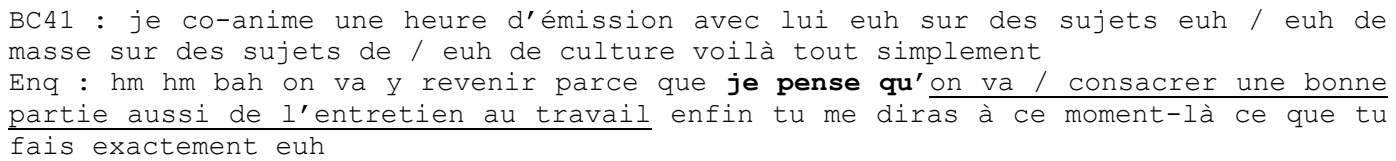

Exemple 20 (ESLO1_ENT_149_je crois que_01:07:27)

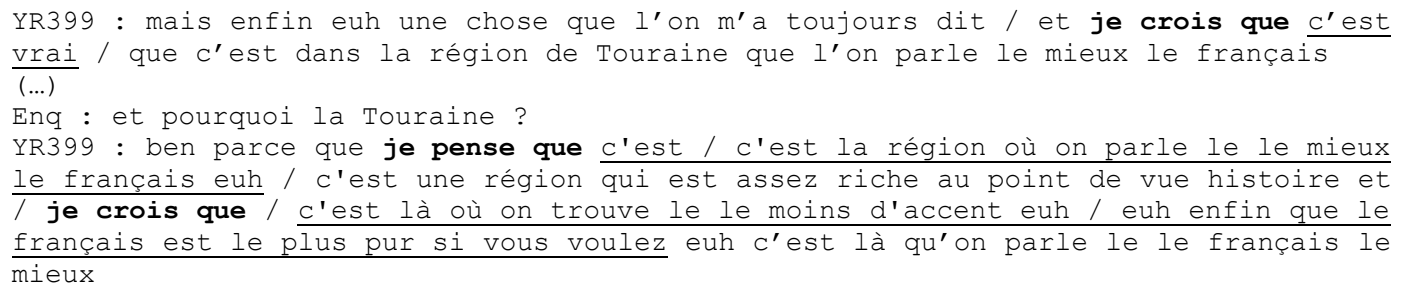

L'analyse de l'exemple 19 correspond à celle de l'exemple 14: il s'agit de valeur de "politesse interactionnelle» déjà étudiée par Apothéloz (2003). Si, pour le locuteur-enquêteur, aucun doute ne subsiste sur le fait qu'une grande partie de l'entretien sera dédiée au travail de l'enquêté - il ne s'agit là ni d'une incertitude ni d'un point de vue - cet énoncé est tout de même modalisé, atténué. La justification de ce procédé d'atténuation d'une certitude par je pense que / je crois que se retrouve dans le concept de la gestion des faces en interaction : en les modulant, l'enquêteur agit en sorte que ses propos ne soient pas perçues comme trop directifs. De cette manière, il préserve sa face dans l'interaction.

Contrairement à l'exemple 19, le suivant est plus complexe. Trois énoncés sont enchâssés par je crois que I je pense que. Le premier - je crois que c'est vrai - correspond à la valeur de modalisation épistémique. Hors contexte, le deuxième - je pense que... - pourrait être analysé comme ayant également la valeur de modalisation : le locuteur exprime son point de vue, ce qui est rendu évident par le présentatif c'est et l'adverbe évaluatif le mieux. Or on apprend dans le contexte gauche que le locuteur tient cette constatation - dans la région de Touraine on parle le meilleur français - d'une source énonciative extérieure - on m'a toujours dit. Ainsi, ce deuxième énoncé est également interprété comme ayant la valeur de certitude atténuée (qui peut être analysée comme ayant été modulée par le point de vue du locuteur, cf. 3.3.), ce qui se confirme dans la suite où le locuteur explicite et argumente ses propos. Dans cette perspective, le troisième énoncé - je crois que c'est là où... - rejoint l'argumentation avancée pour le précédent: le locuteur module, atténue la certitude qu'il accorde au contenu propositionnel de la complétive.

Finalement, on peut dire que cette dernière valeur se manifeste par deux emplois. Le premier tend à relativiser, par politesse interactionnelle, la certitude véhiculée par le contenu propositionnel en complétive. Si dans notre corpus cet emploi est associé à l'enquêteur, c'est en raison de la particularité interactive du genre entretien : comme c'est à l'enquêteur de mener l'entretien en posant des questions, il est plus probable que ce sera lui qui revienne sur du déjà-dit, des évidences qu'il modalise par la suite. Dans d'autres genres interactionnels, cet emploi se caractérise par le seul fait que le contenu propositionnel dans la proposition complétive concerne l'interlocuteur ou la relation interlocutive. Le second emploi diffère du premier sur un point. Si le locuteur est sûr de la vérité ou la validité de l'énoncé dans la complétive, celui-ci n'implique pas l'interlocuteur.

Le tableau suivant récapitule, tout en les exemplifiant, les trois valeurs repérées parmi les occurrences de je crois que / je pense que: 


\begin{tabular}{|c|l|l|}
\hline Valeur & \multicolumn{1}{|c|}{ Propriétés } & \multicolumn{1}{c|}{ Exemple } \\
\hline Argumentation subjective & $\begin{array}{l}\text { Evaluation subjective du } \\
\text { locuteur portant sur le contenu } \\
\text { propositionnel }\end{array}$ & $\begin{array}{l}\text { «je pense que le seul espoir } \\
\text { c'est au niveau des étudiants » }\end{array}$ \\
\hline Incertitude & $\begin{array}{l}\text { Relativisation de la valeur de } \\
\text { vérité du contenu propositionnel, } \\
\text { non-prise en charge }\end{array}$ & $\begin{array}{l}\text { «e crois que c'était Confessions } \\
\text { Intimes (mais je suis pas sûr) » }\end{array}$ \\
\hline Atténuation et certitude & $\begin{array}{l}\text { Atténuation, par politesse, de la } \\
\text { valeur de vérité associée au } \\
\text { contenu propositionnel }\end{array}$ & $\begin{array}{l}\text { «je pense qu'on a dit } \\
\text { l'essentiel }\end{array}$ \\
\hline
\end{tabular}

Tableau 1 : Les trois valeurs de je crois que / je pense que

\subsection{Glissements entre valeurs}

A la suite de la présentation des trois catégories de valeurs accordées aux structures je crois que / je pense $q u e$ en interaction orale, une valeur d'argumentation subjective et deux qui expriment la variation de degré de certitude, une question se pose : peut-on accorder, d'une façon univoque, une valeur à chaque occurrence ou y a-t-il parfois des cas où la frontière entre valeurs serait floue ? De plus, si cela est le cas, est-il possible d'observer une régularité certaine dans ce «continuum » de valeurs?

Observons les exemples suivants :

Exemple 21 (ESLO1_ENT_129_je crois que_00:40:34)

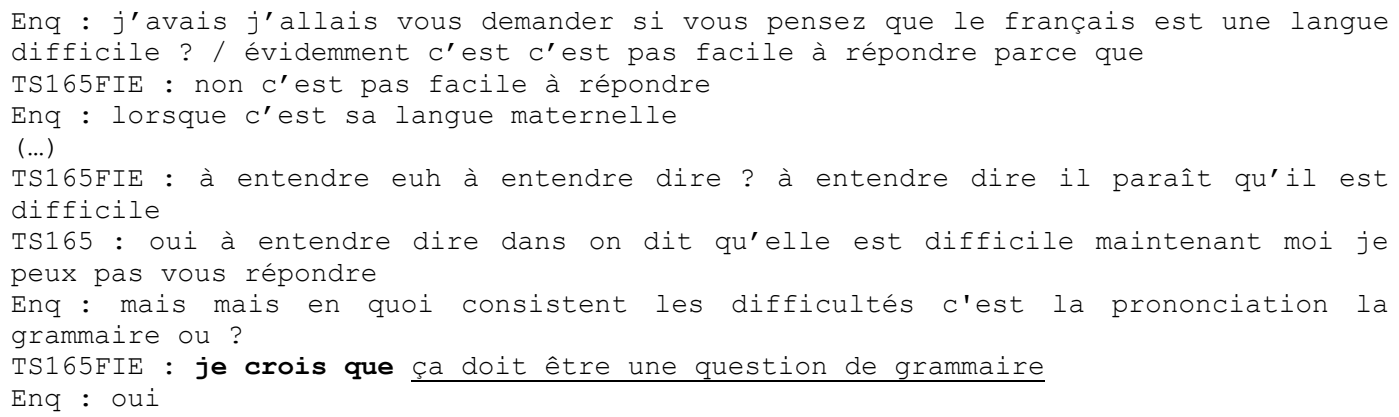

Exemple 22 (ESLO1_ENT_015_je pense que_00:49:50)

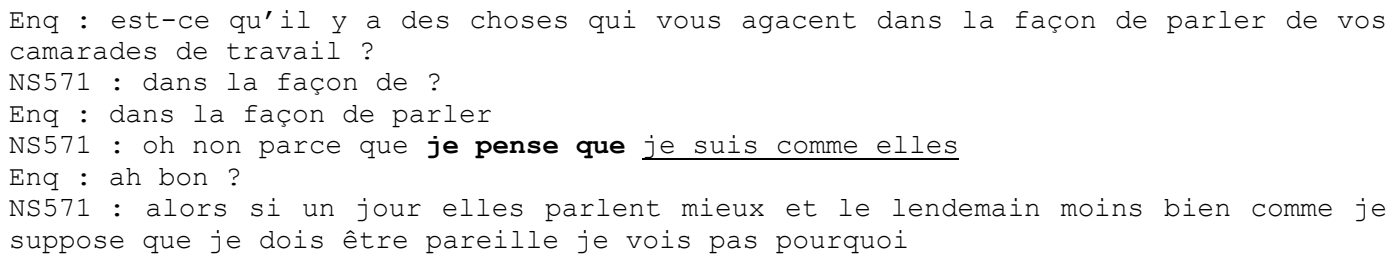

Dans l'exemple 21, la locutrice répond à la question de l'enquêteur par je crois que ça doit être une question de grammaire. L'énoncé dans la complétive exprime son point de vue - il est possible de reformuler la structure principale par à mon avis, selon moi, pour moi ou je me dis. Cependant, le présentatif modalisé par le verbe devoir, à valeur hypothétique, oriente l'interprétation de cet exemple vers une autre direction : l'incertitude de la locutrice quant à la validité de sa réponse. Il semblerait ici que la première valeur se combine avec la deuxième : ce qui est exprimé par je crois que / je pense que, c'est un avis subjectif et l'incertitude quant à sa validité dans la réalité extralinguistique. 
L'exemple 22 présente un autre cas de concomitance de valeurs dans la complétive véhiculées par je crois que / je pense que. La locutrice exprime à la fois son avis sur la question - il lui semble qu'elle parle comme ses collègues - alors qu'en même temps le cotexte droit argumente sa certitude tout en développant l'hypothèse avancée dans l'énoncé enchâssé par je pense que. Dans cet exemple, la première valeur se combine avec la troisième : la proposition principale je pense que accorde à l'énoncé dans la complétive à la fois l'expression du point de vue de la locutrice et l'indication de sa certitude.

Suite à l'observation de ces exemples, il semblerait que se dessinent deux axes dans le système des valeurs véhiculées par je crois que / je pense que. Le premier axe est celui de l'argumentation subjective. Il se rapporte au locuteur : ce n'est pas l'(in)certitude du contenu propositionnel en complétive qui est mise en lumière par la construction à verbe recteur faible, mais la position subjective du locuteur par rapport à ce contenu. Autrement dit, le locuteur exprime son avis subjectif sur ce qu'il exprime par la complétive. Le second axe se rapporte au degré de certitude que le locuteur exprime par rapport au contenu propositionnel de la complétive : il s'agit pour lui d'émettre un commentaire métadiscursif sur la certitude du dire. Les données montrent que ces deux axes peuvent se combiner : la combinatoire est possible entre le premier axe - qui exprime le point de vue subjectif - et l'un des éléments du second - la certitude ou l'incertitude. Or il semble impossible de superposer les valeurs d'un même axe, à savoir l'expression de l'incertitude avec celle de la certitude. Par conséquent, le système de valeurs véhiculées par je crois que / je pense que se dessine en continuum, mais non sans une contrainte distributionnelle : c'est la valeur subjective qui peut se combiner avec celle exprimant l'(in)certitude du dire, puisque les deux types de valeurs opèrent sur deux axes différents. Le premier se rapporte à l'argumentation et à la subjectivité exprimée par le locuteur et le second à l'hypothèse de la validité de l'énoncé dans la complétive selon l'univers de croyance du locuteur.

\subsection{De l'expression de la subjectivité vers la politesse interactionnelle}

Afin de conclure cette partie, nous reviendrons sur quelques observations relatives à l'interrelation entre les trois valeurs repérées parmi les occurrences de structures je crois que / je pense que dans notre corpus. Nous avons pu identifier les caractéristiques propres à chacune des trois valeurs tout en montrant le comportement des structures dans le contexte interactif. De même, l'examen des occurrences du corpus a montré la possibilité de superposer deux catégories de valeurs, la première, exprimant le point de vue, avec l'une des deux qui se rapportent aux divers degrés de la certitude.

Si le parti pris dans l'analyse des valeurs propres aux occurrences dans le corpus consistait à ne pas étudier séparément le sémantisme des je crois que et des je pense que, c'est parce que les trois valeurs identifiées se rapportent aux deux structures de part égale : nous verrons par la suite de quelle manière les deux structures interagissent et surtout ce que peut nous enseigner l'examen de la variation quantitative en micro-diachronie.

La relation entre les trois valeurs montre-t-elle une régularité permettant de les organiser en système ? Il nous semble que le rapport entre les valeurs repérées se cristallise dans l'image de l'interaction, autrement dit relativement à la figure de l'interlocuteur. Le schéma suivant montre le mouvement opéré entre les trois valeurs :

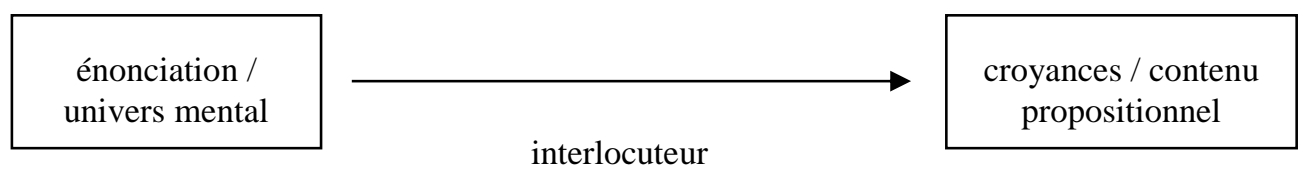

La première valeur, celle d'argumentation subjective, s'associe à la représentation mentale du sujet par lui-même : la figure de l'interlocuteur en est exclue. En exprimant son point de vue, son avis sur l'énoncé dans la complétive, le locuteur reste dans la sphère privée : il s'agit d'un renforcement interne, d'une subjectivation du dire par l'énonciation marquée à la première personne. Comment se manifeste la nonprise en compte de l'interlocuteur dans la revendication de son point de vue ? C'est ce que montre 
l'exemple 16: quand bien même la situation de communication relèverait d'un cadre semi-formel, le locuteur «s'autorise » le mot connerie dans l'expression de son point de vue quant à la construction de nouveaux bâtiments dans une commune orléanaise.

Plus le changement s'opère vers la troisième valeur, celle de l'expression d'une certitude modalisée par politesse, plus est pris en compte le cadre interlocutif, plus l'autre est engagé dans ce que «je » dit. Dans la troisième valeur, ceci est transparent : l'emploi atténuateur de certitude dans un contexte qui inclut explicitement l'interlocuteur engage celui-ci par excellence. C'est la figure de l'interlocuteur et l'image de l'interlocution qui justifient l'utilisation de je crois que / je pense que à valeur d'atténuateur sociointeractionnel. Ainsi en va-t-il du second emploi de la troisième valeur, lorsque le locuteur atténue une certitude qui n'implique pas l'interlocuteur.

La seconde valeur, celle exprimant l'incertitude du locuteur quant au contenu propositionnel de l'énoncé en complétive, engage l'interlocuteur indirectement : en modulant ses propos par je crois que / je pense $q u e$ à valeur d'incertitude, le locuteur préserve sa face en s'autorisant une « marge d'erreur » quant à la validité de l'énoncé qu'il modalise.

Ainsi, le système de valeurs accordées aux structures je crois que / je pense que se manifeste dans le rapport à la situation interlocutive. Plus l'autre est pris en compte, moins le locuteur sera impliqué dans l'expression de son point de vue pour s'orienter davantage vers le contenu du dire et sa modalisation selon un degré de certitude. La variation des valeurs de modalisation est renforcée dans le système de la circulation sociale de la parole, tout en accordant au locuteur la possibilité de revendiquer sa subjectivité en « ignorant » la représentation interlocutive.

\section{De je crois que vers je pense que : remarques micro-diachroniques}

Le tableau suivant met en relation la proportion d'actualisation de chaque structure, aussi bien en microdiachronie, dans l'intervalle de 40 ans, et en synchronie, ce qui permet de comparer les deux structures dans le sous-corpus retenu pour cette étude.

\begin{tabular}{|c|c|c|c|}
\cline { 2 - 4 } \multicolumn{1}{c|}{} & ESLO1 $^{11}$ & ESLO2 & ESLO1 + ESLO2 \\
\hline je crois que & 357 & 117 & 474 \\
\hline je pense que & 130 & 287 & 417 \\
\hline
\end{tabular}

Les résultats de l'approche quantitative appellent trois observations :

- le nombre d'occurrences de chacune des deux structures dans l'ensemble de notre corpus est comparable ;

- d'un point de vue synchronique, je crois que est la structure privilégiée dans le contexte d'ESLO1 et il en va de même pour je pense que dans ESLO2;

- sur quarante ans, il y a une forte hausse de fréquence de la structure je pense que (deux fois plus) alors que, dans la même période, je crois que subit une baisse considérable (trois fois moins). Notons, par ailleurs, que ces mêmes proportions concernent l'ensemble des occurrences de je crois et je pense (c'està-dire les trois types d'emplois confondus) dans ESLO1 et ESLO2. Je crois passe de 620 occurrences dans ESLO1 à 230 dans ESLO2 et je pense de 203 dans ESLO1 à 461 dans ESLO2.

La stabilité d'emploi des deux structures dans notre corpus indépendamment de la période de leur actualisation - 474 occurrences pour je crois que contre 417 occurrences pour je pense que - pourrait conforter l'hypothèse de leur relative interchangeabilité. Or l'examen des proportions des occurrences dans chacun des deux sous-corpus nous laisse concevoir une évolution de la fréquence au profit de je pense que. Nous expliquons ce changement par l'hypothèse suivante : il se peut que le sémantisme de la 
structure je pense que soit en train de se déspécialiser, de progresser vers des emplois en contexte qui, dans ESLO1, étaient notablement associés aux structures avec croire.

Au-delà de ce qui rassemble les deux constructions étudiées - une même échelle de valeurs et des emplois contextuels qui semblent interchangeables - une question reste sans réponse : si les deux structures peuvent permuter aussi bien contextuellement que pragmatiquement, pourquoi alors la langue autorise-telle leur parallélisme ? Nous terminerons notre analyse en signalant ce qui est propre à chaque structure et qui se manifeste dans leur relation en micro-diachronie.

Observons les deux exemples suivants, extraits respectivement d'ESLO1 et d'ESLO2, où je crois que et je pense que s'actualisent successivement.

Exemple 23 (ESLO1_ENT_141_je crois que_01:04:34)

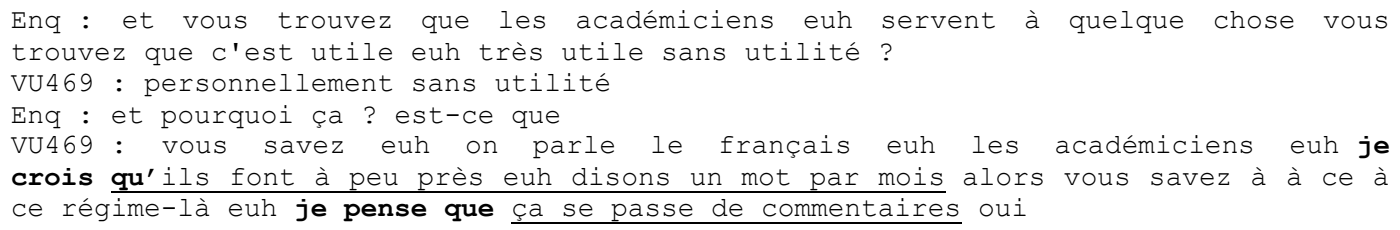

Exemple 24 (ESLO2_ENT_1081_je crois que_00:02:40)

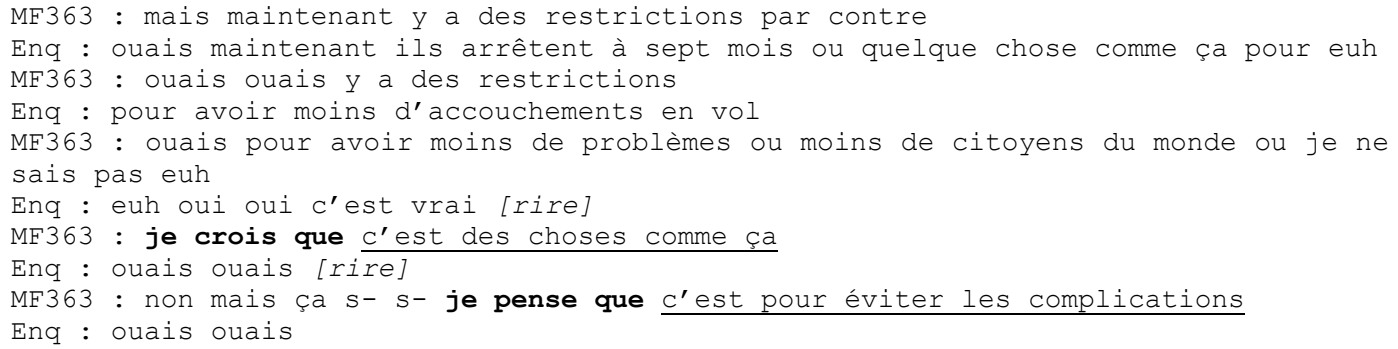

Dans l'exemple 23, le contenu propositionnel du premier énoncé en complétive, régi par je crois que, s'oriente vers un commentaire - subjectif certes - mais relatif à une réalité extérieure au locuteur, commentaire sur le travail de l'Académie française. En revanche, le second énoncé en complétive, régi par je pense que, ne se rapporte qu'à ce qui se présente comme étant propre à la subjectivité du locuteur.

Un emploi successif de je crois que et je pense que est relevé également dans l'exemple 24. Si l'analyse de la première structure je crois que... concorde avec celle de l'exemple précédent - le commentaire du locuteur se rapporte à la réalité objective - ce n'est pas le cas avec l'occurrence de je pense que. On note en effet un même contexte pour je crois que et je pense que. Ce dernier reformule en le suppléant l'énoncé en complétive précédente, «c'est des choses comme ça ». Autrement dit, je crois que et je pense que dans l'exemple 24 ont la même portée : il modalisent une réalité extérieure au locuteur.

Il nous semble que c'est ici qu'opère le changement subi par la structure je pense que, changement sémantique de la portée du verbe dont l'emploi a doublé en l'espace de quarante ans, et qui prend le rôle de je crois que - une structure dont l'amplitude pragmatique est plus importante. Il reste qu'un examen minutieux des données est nécessaire afin de comparer tous les contextes d'émergence des deux structures dans les deux sous-corpus et d'approfondir cette réflexion : à terme, une telle entreprise pourra indiquer d'une manière précise l'échantillonnage des contextes et la portée du changement opérée en micro-diachronie entre les complétives de je crois que et celles de je pense que.

Pourquoi je pense que est-il en train de suppléer je crois que ? Parallèlement à l'affaiblissement du sens de penser, qui d'une représentation mentale du sujet par lui-même se déplace vers tout contexte d'expression de la subjectivité (ce qui lui permet d'étendre ses emplois vers les contextes jusque-là réservés à je crois que), le changement opéré en l'espace de quarante ans s'oriente vers le domaine de la 
«pensée » et au-delà des « croyances ». Rappelons-le, dans l'échantillon des trois valeurs accordées aux deux structures, la valeur propre à l'expression du point de vue, d'une activité mentale, se repère à environ $75 \%$ parmi les occurrences de je pense que alors qu'elle n'atteint qu'environ $45 \%$ dans celles de je crois que. Ainsi, si les deux constructions se rapportent à «l'énonciation qui actualise une certaine forme d'inscription du locuteur en première personne dans son dire » (Bergounioux, $2015: 21$ ), c'est la séquence avec je pense que qui symbolise par excellence le locuteur en tant qu'individu, face à une circulation sociale de croyances. Et on pourrait dire avec Alain (Propos d'un Normand, 15 janvier 1908), «penser, c'est inventer sans croire ». Autrement dit, c'est un acte individuel et individualisant.

Au final, si l'emploi de «je pense que » est en hausse, c'est à la fois grâce à un affaiblissement du sens de penser et à un potentiel changement énonciatif en cours, qui se traduit par l'émergence des marques formelles traduisant l'ipséité du locuteur dans la conception sociale de l'interaction.

\section{Conclusion et perspectives}

Après avoir appréhendé les différents types d'emplois de je crois et je pense (propositionnels et parenthétiques), nous avons proposé une analyse des valeurs sémantico-pragmatiques qu'ils projettent en interaction. A côté des valeurs repérées pour les emplois propositionnels «pleins », nous nous sommes penchées plus spécifiquement sur les valeurs propres aux emplois parenthétiques je crois que / je pense que.

Trois valeurs ont été identifiées : argumentation subjective, incertitude et atténuation-certitude. Ces valeurs forment un système construit sur le degré de prise en compte de l'interlocuteur dont les traces se retrouvent aussi bien dans la complétive qu'en cotexte. Cela nous a amenées à proposer un schéma situant les valeurs de je crois que et je pense que sur une échelle allant de l'univers mental du locuteur vers celui des croyances partagées.

Par ailleurs, l'approche quantitative des données en micro-diachronie a permis d'observer une hausse considérable de la fréquence d'emploi de je pense que au dépens de celle de je crois que. Nous avons interprété ces écarts en termes de déspécialisation sémantico-pragmatique de je pense que qui lui permet de couvrir un éventail d'emplois réservés jusque-là à je crois que. La structure je pense que passe ainsi de l'ombre de je crois que dans ESLO1 au statut de structure privilégiée dans ESLO2.

Un éclairage supplémentaire sur la déspécialisation de je pense que pourra être apporté par un examen des indices contextuels spécifiques ou communs afin d'identifier les « lieux » où cette structure relaie je crois que. De même, si dans cette étude nous avons privilégié une approche descriptive des deux structures « figées », je crois que et je pense que, il ne faudra pas exclure, pour une analyse ultérieure, la variation interne aux propositions parenthétiques, et ce notamment en relation avec les temps verbaux et avec les personnes grammaticales. Bien que la discrimination des emplois des deux structures ait été établie à partir d'éléments sémantico-pragmatiques et interactionnels, une étude de la dimension prosodique ${ }^{12}$ permettra d'enrichir la description des emplois.

Enfin, une analyse contrastive en synchronie des emplois des deux structures par des locuteurs appartenant à des catégories socio-professionnelles différentes permettra de repérer des traces de variation diastratique à l'origine du changement diachronique.

\section{Références bibliographiques}

Abouda, L., Baude, O. (2007a). Constituer et exploiter un grand corpus oral : choix et enjeux théoriques. Le cas des Eslo, in F. Rastier et M. Ballabriga (dir.), Corpus en Lettres et Sciences sociales. Des documents numériques à l'interprétation, Actes du XXVIIe Colloque d'Albi, juillet 2006, 161-168.

Abouda, L., Baude, O. (2007b). Du français fondamental aux Eslo, Les Cahiers de Linguistique de Louvain, vol. 33, 2, 131-146. 
Abouda, L, Skrovec, M. (2015). Du rapport entre formes synthétique et analytique du futur. Étude de la variable modale dans un corpus oral micro-diachronique, Revue de Sémantique et de Pragmatique, 38

Andersen, H.L. (1996). Verbes parenthétiques comme marqueurs discursifs. In Muller, C. (éd.), Dépendance et intégration syntaxique : subordination, coordination, Tübingen : M. Niemeyer, 307-315.

Apothéloz, D. (2003). La rection dite «faible » : grammaticalisation ou différentiel de grammaticité ? Verbum XXV : $3,241-262$.

Baude, O., Dugua, C. (2011). (Re)faire le corpus d'Orléans quarante ans après : quoi de neuf, linguiste ? Corpus, Varia, 10, 99-118.

Benveniste, E. (1966). De la subjectivité dans le langage. Problèmes de linguistique générale, 1, Paris, Gallimard, 258-266.

Bergounioux, G. (2015). Organisation du discours et énonciation en français parlé. Les changements étudiés à partir d'un enregistrement d'ESLO2. Journal of language and culture, Language and information, 10, 1-22.

Blanche-Benveniste, C. (1989). Constructions verbales «en incise» et rection faible des verbes. Recherches sur le français parlé, 9, 53-74.

Doro-Mégy, F. (2008). Etude croisée de think, believe, croire et penser. Linguistique contrastive et traduction, $\mathrm{N}^{\circ}$ spécial, Paris, Ophrys.

Eshkol-Taravella, I., Baude, O., Maurel, D., Hriba, L., Dugua, C., Tellier, I. (2012). Un grand corpus oral « disponible »: le corpus d'Orléans 1968-2012. Ressources linguistiques libres, TAL, 52 :3, 17-46.

Kerbrat-Orecchioni, C. (1980). L'énonciation - De la subjectivité dans le langage, Paris, Armand Colin.

Martin, R. (1988). Croire que p / penser que p. Annexes des cahiers de linguistique hispanique médiévale, 7, Hommage à Bernard Pottier, 547-554.

Mullan, K. (2012). Saying what you think: an analysis of French and Australian English non-native speaker expression of subjectivity, in N. Baumgarten et al. (ed.), Subjectivity in Language and in Discourse, United Kingdom, Emerald Group Publishing Ltd, 193-227.

Mullan, K. (2010). Expressing opinions in French and Australian English Discourse: A semantic and interactional analysis. Amsterdam, John Benjamins.

Mullineaux, L.A., Blanc, M. H. A. (1982). The problem of classifying the population sample in the socio linguistic survey of Orléans (1969) in terms of socio-economic, social and educational categories. Review of Applied Linguistics, 55, 3-37.

Pottier, B., (1983). Le croire dans une représentation sémio-linguistique dynamique, in H. Parret (ed.), On Believing Epistemological and semiotic approaches. De la croyance, Approches épistémologiques et sémiotiques, Berlin, New York, Walter de Gruyter, 267-273.

Urmson, J.O. (1952). Parenthetical verbs. Mind, 61, 480-496.

\footnotetext{
${ }^{1}$ Annoncée auparavant par Benveniste (1966) relativement au verbe croire.

${ }^{2}$ Le corpus est disponible à l'adresse suivante : http://eslo.huma-num.fr/.

${ }^{3}$ Echelle AM, conçue à partir des CSP de l'INSEE de l'époque avec un enrichissement de caractéristiques culturelles et de critères liés à la mobilité géographique, cf. Mullineaux \& Blanc (1982).

${ }^{4}$ Cf. Abouda \& Baude (2007a et b) pour des précisions historiques, épistémologiques et méthodologiques sur ESLO.

${ }^{5}$ En termes de profil de locuteurs, choisis selon les variables de sexe (H/F), d'âge (3 tranches) et de catégorie socioprofessionnelle (5 classes).

${ }^{6}$ A côté des entretiens, le sous-corpus compte à hauteur de $20 \%$ (en termes de nombre d'heures) des enregistrements de repas et de conférences.

${ }^{7}$ Les crochets encadrant des points de suspension marquent la suppression d'une partie du tour de parole du locuteur ou d'une partie du passage de transcription sélectionné.
} 
${ }^{8}$ Les codes locuteurs des enquêteurs ont été uniformisés par «Enq » pour une meilleure lisibilité des exemples.

${ }^{9}$ Les signes \# précédés des chiffres 1 et 2 encadrent des séquences de chevauchement.

${ }^{10}$ (ESLO1_ENT_072_on pense pas_00:24:43)

FZ61: C'est un peu une conséquence de cette civilisation de consommation tout le monde cherche à avoir son frigidaire son auto son week-end euh on ne pense pas beaucoup Enq: oui oui

11 «ESLO1 » et «ESLO2» ne renvoient pas à l'ensemble du corpus mais uniquement aux données appartenant au sous-corpus utilisé.

${ }^{12}$ Cf. Mullan (2010) pour une étude qui prend en compte de manière centrale la prosodie. 